\title{
A New Algorithm for Model Order Reduction of Interval Systems
}

\author{
D. Kranthi Kumar, S.K. Nagar and J.P. Tiwari
}

\begin{abstract}
Mixed method of interval systems is a combination of classical reduction methods and stability preserving methods of interval systems. This paper proposed a new method for model order reduction of systems with uncertain parameters. The bounds on the uncertain parameters are known a priori. Two separate methods are used for finding parameters of the numerator and denominator. The numerator parameters are obtained by either of these methods such as differentiation method, factor division method, cauer second form, moment matching method or Pade approximation method. The denominator is obtained by the differentiation method in all the cases. A numerical example has been discussed to illustrate the procedures. From the above mixed methods, differentiation method and cauer second form as resulted in better approximation when compared with other methods. The errors between the original higher order and reduced order models have also been highlighted to support the effectiveness of the proposed methods.
\end{abstract}

Keywords--- Cauer Second Form, Differentiation Method, Factor Division, Interval Systems, Moment Matching, Mixed Method, Pade Approximation

\section{INTRODUCTION}

$\mathrm{A}$ PPROXIMATION of higher order systems to lower order models facilitates simulation and design over the complex models. There has been a tremendous growth in the research area of model order reduction, resulting in a development of variety of techniques. Also, engineering and science designs involve uncertainty specified in a number of ways, as convex or fuzzy descriptions to varying degree, inclining a must study to estimate the upper and lower bounds of the systems for the proper examination. Thus, systems with constant coefficients but uncertain within finite range, is classified under interval systems. Over the years, analysis, stability and transients of interval systems have attracted the

D. Kranthi Kumar, Research Scholar, Department of Electrical Engineering, IIT-BHU, Varanasi, U.P, India. E-mail:kranthi.kumar.eee08@itbhu.ac.in.

S. K. Nagar, Professor, Department of Electrical Engineering, IIT-BHU, Varanasi, U.P, India. E-mail:sknagar.eee@itbhu.ac.in.

J. P. Tiwari, Professor, Department of Electrical Engineering, IIT-BHU, Varanasi, U.P, India. E-mail:jptewari@bhu.ac.in

DOI: 10.9756/BIJDM.10131 attention of researchers, provided only fewer literatures available. From the list, Eigen value problem is best suited.

Considerable attention can be observed by the available amount of literature, commencing from the basics of aggregation method [1] for reduced order model. Some other classical reduction methods include moment matching, Pade approximation and Continued fraction expansion methods [29] and many more. These methods do not guarantee stability, was the only drawback. To overcome this problem, stability preservation methods (SPM), as Routh approximation using $\alpha$ and $\beta$ table [3], optimal hankel norm approximation [6] etc were used for fixed parameter systems. However, many practical system models, like flexible manipulator system or nuclear reactor systems with large area of parameter uncertainties, lead to another area of research with the pioneering circle of Kharitonov method [10], where the stability of reduced order model was of great concern.

Literatures have also exposed interval systems to study their stability and the transient analysis [10-11]. For reduction of continuous interval systems, Routh approximation [12], $\gamma-\delta$ Routh approximation [13], and also to reduce the complexity of calculations a simple direct method using $\gamma$ table [14] have also been proposed. Dolgin and Zeheb [15], [17] modified the Bandoypadhyay [13] method and suggested new mixed method to overcome the instability of the system. Unfortunately, Hwang et.al [16] commented on Dolgin's method and proved the failure of stability. Apart from these several other mixed methods [18-27] were proposed recently to reduce the complexity and to increase the accuracy of the system. To find the Eigen values to the uncertain systems has its own history. Finding poles for uncertain systems [19-22] is not computationally simple, but these mixed methods are giving stable reduced order systems. As an alternative we wish to point out that there is a large variety of ways to reduce the order of a specified polynomial. Of these methods we have chosen to study differentiation. an operation well known to all persons with even a trivial contact with mathematical analysis.

Differentiation method [29] was proposed by Gutman for fixed coefficients and Author has extended this method to interval systems and also proposes some new mixed methods.

In this paper, some mixed methods for model order reduction of interval systems is proposed. The denominator of the interval reduced model is obtained by differentiation method. The numerator is obtained by different mixed methods, such as direct truncation of differentiation, factor division, Cauer second form, Moment matching method and Pade approximation method. These methods ensure that the 
reduced order model is stable if the higher order uncertain system is asymptotically stable. The outline of this paper is as follows: Section II contains problem statement; Section III contains proposed method and integral square error. Numerical example is presented in section IV and conclusions in section $\mathrm{V}$.

\section{PRoblem StATEMENT} be:

Let the transfer function of a higher order interval systems

$G_{n}(s)=\frac{\left[c_{21}^{-}, c_{21}^{+}\right]+\left[c_{22}^{-}, c_{22}^{+}\right] s+\ldots .+\left[c_{2 n}^{-}, c_{2 n}^{+}\right] s^{n-1}}{\left[c_{11}^{-}, c_{11}^{+}\right]+\left[c_{12}^{-}, c_{12}^{+}\right] s+\ldots .+\left[c_{1, n+1}^{-}, c_{1, n+1}^{+}\right] s^{n}}=\frac{N(s)}{D(s)}$

The reduced order model of a transfer function be considered as

$R_{k}(s)=\frac{\left[d_{21}^{-}, d_{21}^{+}\right]+\left[d_{22}^{-}, d_{22}^{+}\right] s+\ldots .+\left[d_{2 k}^{-}, d_{2 k}^{+}\right] s^{k-1}}{\left[d_{11}^{-}, d_{11}^{+}\right]+\left[d_{12}^{-}, d_{12}^{+}\right] s+\ldots .+\left[d_{1, k+1}^{-}, d_{1, k+1}^{+}\right] s^{k}}=\frac{N_{k}(s)}{D_{k}(s)}$

The rules of the interval arithmetic have been defined in [28], and are presented below.

Let $[\mathrm{e}, \mathrm{f}]$ and $[\mathrm{g}, \mathrm{h}]$ be two intervals.

Addition:

$[e, f]+[g, h]=[e+g, f+h]$

Subtraction:

$[e, f]-[g, h]=[e-h, f-g]$

Multiplication:

$[e, f] \times[g, h]=[\operatorname{Min}(e g, e h, f g, f h), \operatorname{Max}(e g, e h, f g, f h)]$

Division:

$\frac{[e, f]}{[g, h]}=[e, f] \times\left[\frac{1}{h}, \frac{1}{g}\right]$

\section{PROPOSED Methods}

The proposed method consists of the following steps for obtaining reduced order model.

Step 1: Determination of the denominator polynomial of the $k^{\text {th }}$ order reduced model as given in Eq (2) by using differentiation method:

$$
D_{k}(s)=\frac{1}{s} D\left(\frac{1}{s}\right)
$$

Differentiate the above equation (2) into (n-k) times

$$
D_{k}(s)=\left[d_{11}^{-}, d_{11}^{+}\right]+\left[d_{12}^{-}, d_{12}^{+}\right] s+\ldots .+\left[d_{1, k+1}^{-}, d_{1, k+1}^{+}\right] s^{k}
$$

Case 1: Determination of the numerator coefficients of the $k^{\text {th }}$ order reduced model by using differentiation method:

$$
N_{k}(s)=\frac{1}{s} N\left(\frac{1}{s}\right)
$$

Differentiate the numerator of Eq (2) into (n-k) times

$$
N_{k}(s)=\left[d_{21}^{-}, d_{21}^{+}\right]+\left[d_{22}^{-}, d_{22}^{+}\right] s+\ldots .+\left[d_{2, k+1}^{-}, d_{2, k+1}^{+}\right] s^{k-1}
$$

Case 2: Determination of the denominator polynomial of the $k^{\text {th }}$ order reduced model by using factor division method.
Any method of reduction which relies upon calculating the reduced denominator first and then the numerator, where $D_{k}(s)$ has already been calculated.

$$
\begin{aligned}
& G_{n}(s)=\frac{N(s) D_{k}(s) / D(s)}{D(s)} \\
& N(s) D_{k}(s)=\left[u_{11}^{-}, u_{11}^{+}\right]+\left[u_{12}^{-}, u_{12}^{+}\right] s+\ldots .+\left[u_{1, k-1}^{-}, u_{1, k-1}^{+}\right] s^{k-1} \\
& \frac{N(s) D_{k}(s)}{D(s)}=\frac{\left[u_{11}^{-}, u_{11}^{+}\right]+\left[u_{12}^{-}, u_{12}^{+}\right] s+\ldots .+\left[u_{1, k-1}^{-}, u_{1, k-1}^{+}\right] s^{k-1}}{\left[d_{11}^{-}, d_{11}^{+}\right]+\left[d_{12}^{-}, d_{12}^{+}\right] s+\ldots .+\left[d_{1, k+1}^{-}, d_{1, k+1}^{+}\right] s^{k}}
\end{aligned}
$$

Therefore,

$$
\begin{aligned}
& {\left[\alpha_{11}^{-}, \alpha_{11}^{+}\right]=\frac{\left[u_{11}^{-}, u_{11}^{+}\right]}{\left[d_{11}^{-}, d_{11}^{+}\right]}\left\{\begin{array}{l}
{\left[u_{11}^{-}, u_{11}^{+}\right]\left[u_{12}^{-}, u_{12}^{+}\right] \ldots . .} \\
{\left[d_{11}^{-}, d_{11}^{+}\right]\left[d_{12}^{-}, d_{12}^{+}\right] \ldots .}
\end{array}\right\}} \\
& {\left[\alpha_{12}^{-}, \alpha_{12}^{+}\right]=\frac{\left[r_{11}^{-}, r_{11}^{+}\right]}{\left[d_{11}^{-}, d_{11}^{+}\right]}\left\{\begin{array}{l}
{\left[r_{11}^{-}, r_{11}^{+}\right]\left[r_{12}^{-}, r_{12}^{+}\right] \ldots .} \\
{\left[d_{11}^{-}, d_{11}^{+}\right]\left[d_{12}^{-}, d_{12}^{+}\right] \ldots .}
\end{array}\right\}} \\
& {\left[\alpha_{13}^{-}, \alpha_{13}^{+}\right]=\frac{\left[s_{11}^{-}, s_{11}^{+}\right]}{\left[d_{11}^{-}, d_{11}^{+}\right]}\left\{\begin{array}{l}
{\left[s_{11}^{-}, s_{11}^{+}\right]\left[s_{12}^{-}, s_{12}^{+}\right] \ldots . .} \\
{\left[d_{11}^{-}, d_{11}^{+}\right]\left[d_{12}^{-}, d_{12}^{+}\right] \ldots . .}
\end{array}\right\}}
\end{aligned}
$$

..................

$$
\begin{aligned}
& {\left[\alpha_{1, k-2}^{-}, \alpha_{1, k-2}^{+}\right]=\frac{\left[x_{11}^{-}, x_{11}^{+}\right]}{\left[d_{11}^{-}, d_{11}^{+}\right]}\left\{\begin{array}{l}
{\left[x_{11}^{-}, x_{11}^{+}\right]\left[x_{12}^{-}, x_{12}^{+}\right]} \\
{\left[d_{11}^{-}, d_{11}^{+}\right]\left[d_{12}^{-}, d_{12}^{+}\right]}
\end{array}\right\}} \\
& \left.\left[\alpha_{1, k-1}^{-}, \alpha_{1, k-1}^{+}\right]=\frac{\left[y_{11}^{-}, y_{11}^{+}\right]}{\left[d_{11}^{-}, d_{11}^{+}\right]}\right]\left\{\begin{array}{l}
{\left[y_{11}^{-}, y_{11}^{+}\right]} \\
{\left[d_{11}^{-}, d_{11}^{+}\right]}
\end{array}\right\}
\end{aligned}
$$

Where

$$
\begin{aligned}
& {\left[r_{1, i}^{-}, r_{1, i}^{+}\right]=\left[u_{1, i+1}^{-}, u_{1, i+1}^{+}\right]-\left[\alpha_{11}^{-}, \alpha_{11}^{+}\right]\left[d_{1, i+1}^{-}, d_{1, i+1}^{+}\right] ; \mathrm{i}=0,1,2, \ldots, \mathrm{k}-2} \\
& {\left[s_{1, i}^{-}, s_{1, i}^{+}\right]=\left[r_{1, i+1}^{-}, r_{1, i+1}^{+}\right]-\left[\alpha_{12}^{-}, \alpha_{12}^{+}\right]\left[d_{1, i+1}^{-}, d_{1, i+1}^{+}\right] ; \mathrm{i}=0,12, \ldots, \mathrm{k}-3} \\
& {\left[y_{1, i}^{-}, y_{1, i}^{+}\right]=\left[x_{11}^{-}, x_{11}^{+}\right]-\left[\alpha_{1, k-2}^{-}, \alpha_{1, k-2}^{+}\right]\left[d_{11}^{-}, d_{11}^{+}\right]}
\end{aligned}
$$

The reduced transfer function given by

$R_{k}(s)=\frac{\left[\alpha_{11}^{-}, \alpha_{11}^{+}\right]+\left[\alpha_{12}^{-}, \alpha_{12}^{+}\right] s+\ldots . .+\left[\alpha_{1, k-1}^{-}, \alpha_{1, k-1}^{+}\right] s^{k-1}}{D_{k}(s)}$

Where

$\left[\alpha_{11}^{-}, \alpha_{11}^{+}\right]=\left[d_{21}^{-}, d_{21}^{+}\right]$
$\left[\alpha_{12}^{-}, \alpha_{12}^{+}\right]=\left[d_{22}^{-}, d_{22}^{+}\right]$
$\ldots \ldots \ldots \ldots \ldots$.
$\left[\alpha_{1, k-1}^{-}, \alpha_{1, k-1}^{+}\right]=\left[d_{2 k}^{-}, d_{2 k}^{+}\right]$

Case 3: Determination of the denominator polynomial of the $k^{\text {th }}$ order reduced model by using Cauer second form:

Coefficient values from Cauer second form $\left[h_{i}^{-}, h_{i}^{+}\right] \quad(i=1,2$ , $3 \ldots \mathrm{k}$ ) are evaluated by forming Routh array as

$$
\begin{aligned}
& {\left[h_{1}^{-}, h_{1}^{+}\right]=\frac{\left[c_{11}^{-}, c_{11}^{+}\right]}{\left[c_{21}^{-}, c_{21}^{+}\right]}\left\{\begin{array}{l}
{\left[c_{11}^{-}, c_{11}^{+}\right]\left[c_{12}^{-}, c_{12}^{+}\right] \ldots . .} \\
{\left[c_{21}^{-}, c_{21}^{+}\right]\left[c_{22}^{-}, c_{22}^{+}\right] \ldots .}
\end{array}\right\}} \\
& {\left[h_{2}^{-}, h_{2}^{+}\right]=\frac{\left[c_{21}^{-}, c_{21}^{+}\right]}{\left[c_{31}^{-}, c_{31}^{+}\right]}\left\{\begin{array}{l}
{\left[c_{21}^{-}, c_{21}^{+}\right]\left[c_{22}^{-}, c_{22}^{+}\right] \ldots . .} \\
{\left[c_{31}^{-}, c_{31}^{+}\right]\left[c_{32}^{-}, c_{32}^{+}\right] \ldots .}
\end{array}\right\}} \\
& {\left[h_{3}^{-}, h_{3}^{+}\right]=\frac{\left[c_{31}^{-}, c_{31}^{+}\right]}{\left[c_{41}^{-}, c_{41}^{+}\right]}\left\{\begin{array}{l}
{\left[c_{31}^{-}, c_{31}^{+}\right]\left[c_{32}^{-}, c_{32}^{+}\right] \ldots . .} \\
{\left[c_{41}^{-}, c_{41}^{+}\right]\left[c_{42}^{-}, c_{42}^{+}\right] \ldots .}
\end{array}\right\}}
\end{aligned}
$$

The first two rows are copied from the original system numerator and denominator coefficients and rest of the elements are calculated by using well known Routh algorithm. 
$\left[c_{1, j}^{-}, c_{1, j}^{+}\right]=\left[c_{i-2, j+1}^{-}, c_{i-2, j+1}^{+}\right]-\left[h_{i-2}^{-}, h_{i-2}^{+}\right]\left[c_{i-1, j+1}^{-}, c_{i-1, j+1}^{+}\right]$

Where $\mathrm{i}=3,4, \ldots$ and $\mathrm{j}=1,2, \ldots$.

$\left[h_{i}^{-}, h_{i}^{+}\right]=\frac{\left[c_{i, 1}^{-}, c_{i, 1}^{+}\right]}{\left[c_{i+1,1}^{-}, c_{i+1,1}^{+}\right]}$

The coefficient values of $\left[d_{i j}^{-}, d_{i j}^{+}\right](\mathrm{j}=1,2, \ldots . .(\mathrm{k}+1))$ and Cauer quotients $\left[h_{i}^{-}, h_{i}^{+}\right](\mathrm{i}=1.2, \ldots \mathrm{k})$ are matched for finding the coefficients of numerator of the reduced model $R_{k}(s)$.

The inverse Routh array is constructed as

$\left[d_{i+1,1}^{-}, d_{i+1,1}^{+}\right]=\frac{\left[d_{i, 1}^{-}, d_{i, 1}^{+}\right]}{\left[h_{i}^{-}, h_{i}^{+}\right]}$

where $\mathrm{i}=1,2, \ldots, \mathrm{k}$ and $\mathrm{k} \leq \mathrm{n}$.

Also,

$\left[d_{i+1, j+1}^{-}, d_{i+1, j+1}^{+}\right]=\frac{\left[d_{i, j+1}^{-}, d_{i, j+1}^{+}\right]-\left[d_{i+2, j}^{-}, d_{i+2, j}^{+}\right]}{\left[h_{i}^{-}, h_{i}^{+}\right]}$

where $\mathrm{i}=1.2, \ldots,(\mathrm{k}-\mathrm{j})$ and $\mathrm{j}=1,2, \ldots,(\mathrm{k}-1)$

Using the above equations, the numerator coefficients of the reduced model will be obtained.

Case 4: Determination of the numerator polynomial of the $(k-1)^{\text {th }}$ order reduced model by using Moment matching method.

Equation (1) is modifed and written as (17)

$G_{n}(s)=\frac{\left[b_{n}^{-}, b_{n}^{+}\right]+\left[b_{n-1}^{-}, b_{n-1}^{+}\right] s+\ldots .+\left[b_{1}^{-}, b_{1}^{+}\right] s^{n-1}}{\left[a_{n}^{-}, a_{n}^{+}\right]+\left[a_{n-1}^{-}, a_{n-1}^{+}\right] s+\ldots . .+\left[a_{0}^{-}, a_{0}^{+}\right] s^{n}}=\frac{N(s)}{D(s)}$

The higher order transfer function $G_{n}(s)$ may be expanded in a series of positive powers of a following

$G_{n}(s)=\left[c_{0}^{-}, c_{0}^{+}\right]+\left[c_{1}^{-}, c_{1}^{+}\right] s+\left[c_{2}^{-}, c_{2}^{+}\right]+\ldots$.

To calculate the coefficients $\left[c_{i}^{-}, c_{i}^{+}\right]$by using the following method

$$
\begin{array}{r}
\text { Set }\left[c_{0}^{-}, c_{0}^{+}\right]=\frac{\left[b_{n}^{-}, b_{n}^{+}\right]}{\left[a_{n}^{-}, a_{n}^{+}\right]} \text {and for } \mathrm{i}>0 \text { and } \\
{\left[c_{i}^{-}, c_{i}^{+}\right]=\frac{1}{\left[a_{n}^{-}, a_{n}^{+}\right]}\left[\left[b_{n-i}^{-}, b_{n-i}^{+}\right]-\sum_{j=1}^{i}\left[a_{n-j}^{-}, a_{n-j}^{+}\right]\left[c_{i-j}^{-}, c_{i-j}^{+}\right]\right]}
\end{array}
$$

To find the reduced order using the moments

$$
\left[\begin{array}{c}
{\left[v_{1}^{-}, v_{1}^{+}\right]} \\
{\left[v_{2}^{-}, v_{2}^{+}\right]}
\end{array}\right]=\left[\begin{array}{cc}
{\left[w_{11}^{-}, w_{11}^{+}\right]} & {\left[w_{11}^{-}, w_{11}^{+}\right]} \\
{\left[w_{21}^{-}, w_{21}^{+}\right]} & {\left[w_{21}^{-}, w_{21}^{+}\right]}
\end{array}\right]\left[\begin{array}{c}
{\left[f_{1}^{-}, f_{1}^{+}\right]} \\
0
\end{array}\right]+\left[\begin{array}{c}
{\left[f_{2}^{-}, f_{2}^{+}\right]} \\
0
\end{array}\right]
$$

Where

$$
\begin{aligned}
& {\left[v_{1}^{-}, v_{1}^{+}\right]=\left[\begin{array}{llll}
{\left[c_{0}^{-}, c_{0}^{+}\right]} & {\left[c_{1}^{-}, c_{1}^{+}\right]} & \ldots \ldots \ldots . . & {\left[c_{m}^{-}, c_{m}^{+}\right.}
\end{array}\right]^{t}} \\
& {\left[v_{2}^{-}, v_{2}^{+}\right]=\left[\begin{array}{llll}
{\left[c_{m+1}^{-}, c_{m+1}^{+}\right]} & {\left[c_{m+2}^{-}, c_{m+2}^{+}\right]} & \ldots \ldots \ldots . . . & {\left[c_{m+r}^{-}, c_{m+r}^{+}\right.}
\end{array}\right]^{t}} \\
& {\left[f_{1}^{-}, f_{1}^{+}\right]=\left[\left[B_{12}^{-}, B_{12}^{+}\right] \quad\left[B_{13}^{-}, B_{13}^{+}\right] \quad \ldots \ldots \ldots . . . \quad\left[B_{1, r+1}^{-}, B_{1, r+1}^{+}\right]\right]^{t}} \\
& {\left[f_{2}^{-}, f_{2}^{+}\right]=\left[\begin{array}{llll}
{\left[B_{21}^{-}, B_{21}^{+}\right]} & {\left[B_{22}^{-}, B_{22}^{+}\right]} & \ldots \ldots \ldots . . & {\left[B_{2, m+1}^{-}, B_{2, m+1}^{+}\right.}
\end{array}\right]^{t}}
\end{aligned}
$$

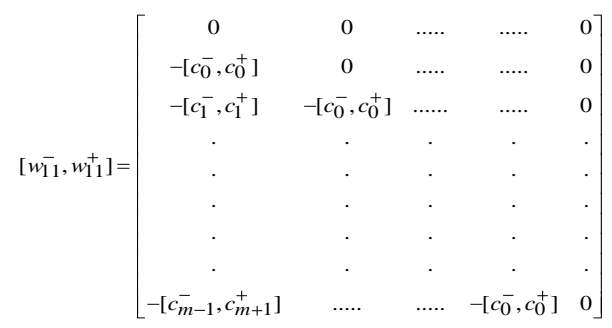

$\left[w_{21}^{-}, w_{21}^{+}\right]=\left[\begin{array}{cccccc}-\left[c_{m}^{-}, c_{m}^{+}\right] & -\left[c_{m-1}^{-}, c_{m-1}^{+}\right] & \ldots . . & -\left[c_{0}^{-}, c_{0}^{+}\right] & \ldots . . & 0 \\ -\left[c_{m+1}^{-}, c_{m+1}^{+}\right] & -\left[c_{m}^{-}, c_{m}^{+}\right] & \ldots . . & -\left[c_{1}^{-}, c_{1}^{+}\right] & \ldots . . & 0 \\ \cdot & \cdot & . & . & . & . \\ \cdot & \cdot & . & . & . & . \\ \cdot & \cdot & . & . & . & . \\ -\left[c_{m+r-1}^{-}, c_{m+r-1}^{+}\right] & -\left[c_{m+r-2}^{-}, c_{m+r-2}^{+}\right] & \ldots . . & \ldots . & \ldots . . & -\left[c_{m}^{-}, c_{m}^{+}\right]\end{array}\right]$

$$
\left[w_{22}^{-}, w_{22}^{+}\right]=\left[\begin{array}{cccccc}
0 & 0 & \ldots . & \ldots . . & \ldots . . & 0 \\
-\left[c_{0}^{-}, c_{0}^{+}\right] & 0 & \ldots . . & \ldots . & \ldots . . & 0 \\
\cdot & . & . & \cdot & \cdot & . \\
\ldots . & \ldots . . & \ldots . . & -\left[c_{0}^{-}, c_{0}^{+}\right] & 0 & 0 \\
\ldots . . & \ldots & \ldots . . & -\left[c_{1}^{-}, c_{1}^{+}\right] & -\left[c_{0}^{-}, c_{0}^{+}\right] & 0
\end{array}\right]
$$

$$
\left[w_{12}^{-}, w_{12}^{+}\right]=[0]
$$

Then unknown parameters are given by

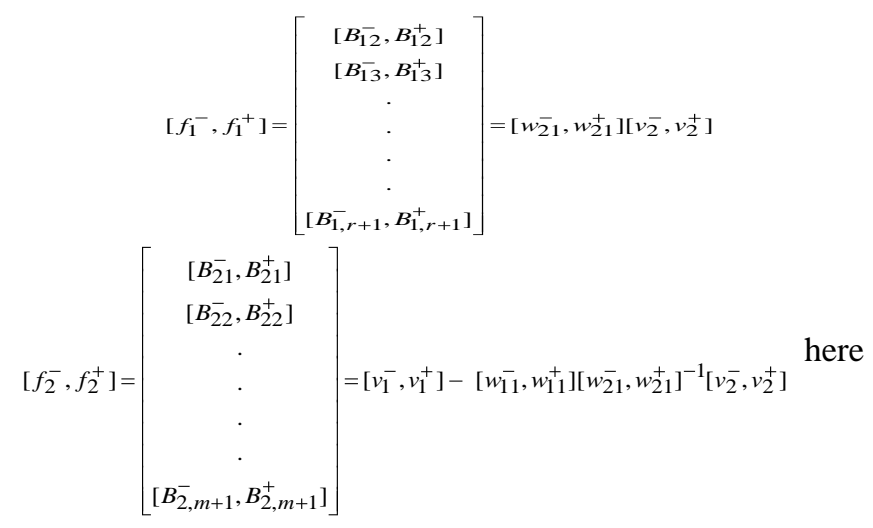

$\left[w_{21}^{-}, w_{21}^{+}\right]$is non singular.

Therefore the reduced transfer function in general form is

$$
R_{k}(s)=\frac{\left[B_{21}^{-}, B_{21}^{+}\right]+\left[B_{22}^{-}, B_{22}^{+}\right] s+\ldots .}{[1,1]+\left[B_{12}^{-}, B_{12}^{+}\right] s+\left[B_{12}^{-}, B_{12}^{+}\right] s^{2}+\ldots .}
$$

Case 5: Determination of the numerator polynomial of the $(k-1)^{\text {th }}$ order reduced model by using Pade approximation method [12].

$$
\begin{aligned}
& \frac{\left[c_{21}^{-}, c_{21}^{+}\right]+\left[c_{22}^{-}, c_{22}^{+}\right] s+\ldots .+\left[c_{2 n}^{-}, c_{2 n}^{+}\right] s^{n-1}}{\left[c_{11}^{-}, c_{11}^{+}\right]+\left[c_{12}^{-}, c_{12}^{+}\right] s+\ldots .+\left[c_{1, n+1}^{-}, c_{1, n+1}^{+}\right] s^{n}}= \\
& \frac{\left[d_{21}^{-}, d_{21}^{+}\right]+\left[d_{22}^{-}, d_{22}^{+}\right] s+\ldots .+\left[d_{2 k}^{-}, d_{2 k}^{+}\right] s^{k-1}}{\left[d_{11}^{-}, d_{11}^{+}\right]+\left[d_{12}^{-}, d_{12}^{+}\right] s+\ldots .+\left[d_{1, k+1}^{-}, d_{1, k+1}^{+}\right] s^{k}} \\
& \quad \text { Where } \\
& {\left[c_{21}^{-}, c_{21}^{+}\right]\left[d_{11}^{-}, d_{11}^{+}\right]=\left[d_{21}^{-}, d_{21}^{+}\right]\left[c_{11}^{-}, c_{11}^{+}\right]}
\end{aligned}
$$


$\left[c_{21}^{-}, c_{21}^{+}\right]\left[d_{12}^{-}, d_{12}^{+}\right]+\left[c_{22}^{-}, c_{22}^{+}\right]\left[d_{11}^{-}, d_{11}^{+}\right]=$

$\left[d_{21}^{-}, d_{21}^{+}\right]\left[c_{12}^{-}, c_{12}^{+}\right]+\left[d_{22}^{-}, d_{22}^{+}\right]\left[c_{11}^{-}, c_{11}^{+}\right]$

$\ldots \ldots \ldots \ldots=\ldots \ldots$

\section{A. Integral Square Error}

The integral square error (ISE) between the transient responses of higher order system (HOS) and Lower order system (LOS) is determined to compare different approaches of model reduction. This is given by

$$
I S E=\int_{0}^{\infty}\left[y(t)-y_{r}(t)\right]^{2}
$$

where, $y(t)$ and $y_{r}(t)$ are the unit step responses of original system $G_{n}(s)$ and reduced order system $R_{k}(s)$.

\section{NUMERICAL EXAMPLE}

This section includes example to illustrate the method.

Example: Consider a third order system described by the transfer function [13]

$$
G_{3}(s)=\frac{[2,3] s^{2}+[17.5,18.5] s+[15,16]}{[2,3] s^{3}+[17,18] s^{2}+[35,36] s+[20.5,21.5]}
$$

Method 1: Reduction by differentiation method.

For getting the second order model, denominator and numerator is obtained by using differentiation method

Number of times to be differentiated is $n-k=3-2=1$.

$$
\begin{gathered}
D_{2}(s)=[4.25,4.5] s^{2}+[17.5,18] s+[15.375,16.125] \\
N_{2}(s)=[5.8333,6.1667] s+[10.0005,10.6672]
\end{gathered}
$$

The reduced second order model $R_{2}(s)$ is given as

$$
R_{2}(s)=\frac{[5.8333,6.1667] s+[10.0005,10.6672]}{[4.25,4.5] s^{2}+[17,18] s+[15.375,16.125]}
$$

The step response of original and reduced second order are shown in figure 1

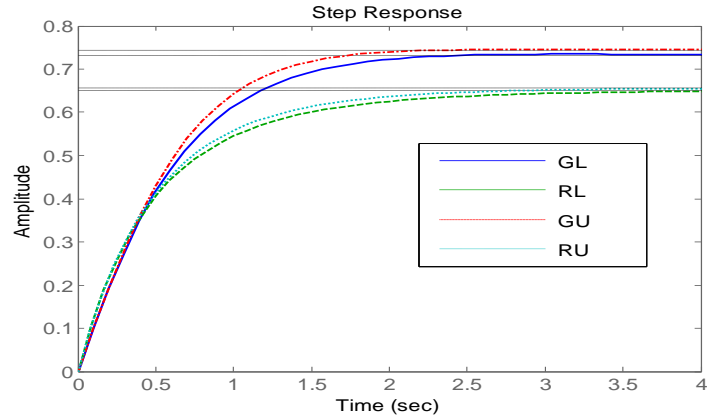

Figure 1: Step Response of Original Model and ROM by Differentiation Method

Method 2: Reduction by differentiation and factor division method

For getting the second order model, denominator by using differentiation method

Number of times to be differentiated is (n-k) times

$\mathrm{n}=$ order of the higher order system $=3$

$\mathrm{k}=$ order of lower order system $=2$

$$
D_{2}(s)=[4.25,4.5] s^{2}+[17.5,18] s+[15.375,16.125]
$$

Numerator is reduced by factor division method

Step 1: Using the factor division method

$$
\frac{N(s) D_{2}(s)}{D(s)}=\frac{[230.625,258]+[531.0562,586.3125] s+\ldots}{[20.5,21.5]+[35,36] s+\ldots}
$$

Step2: Finding the values of $\left[\alpha_{11}^{-}, \alpha_{11}^{+}\right]$and $\left[\alpha_{12}^{-}, \alpha_{12}^{+}\right]$

$\left[\alpha_{11}^{-}, \alpha_{11}^{+}\right]=[10.7267,12.5854]$

$\left[\alpha_{12}^{-}, \alpha_{12}^{+}\right]=[3.4809,10.7884]$

$N_{2}(s)=[3.4809,10.7884] s+[10.7267,12.5854]$

Step3: The reduced transfer function is

$R_{2}(s)=\frac{[3.4809,10.7884] s+[10.7267,12.5854]}{[4.25,4.5] s^{2}+[17,18] s+[15.375,16.125]}$

The step response of orginal and reduced second order are shown in figure 2

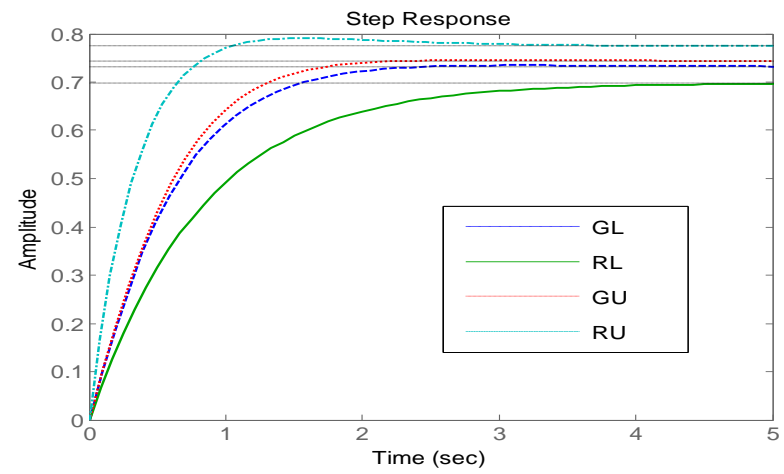

Figure 2: Step Response of Original Model and ROM by Differentiation and Factor Division Method

Method 3: Reduction by differentiation and Cauer second form method

Step 1: Using the Cauer second form as described in the section 3 , it is obtained as

$$
\begin{aligned}
& {\left[h_{1}^{-}, h_{1}^{+}\right]=[1.2812,1.4333]} \\
& {\left[h_{2}^{-}, h_{2}^{+}\right]=[1.1046,1.8859]} \\
& {\left[d_{21}^{-}, d_{21}^{+}\right]=[10.7271,12.5856]} \\
& {\left[d_{22}^{-}, d_{22}^{+}\right]=[4.2604,9.6099]}
\end{aligned}
$$

Step 2: Numerator of second order system is written as $N_{2}(s)=[4.2604,9.6099] s+[10.7271,12.5856]$

Step 3: The reduced order model $R_{2}(s)$ is given as

$R_{2}(s)=\frac{[4.2604,9.6099] s+[10.7271,12.5856]}{[4.25,4.5] s^{2}+[17,18] s+[15.375,16.125]}$

The step response of original and reduced second order is shown in figure 3 . 


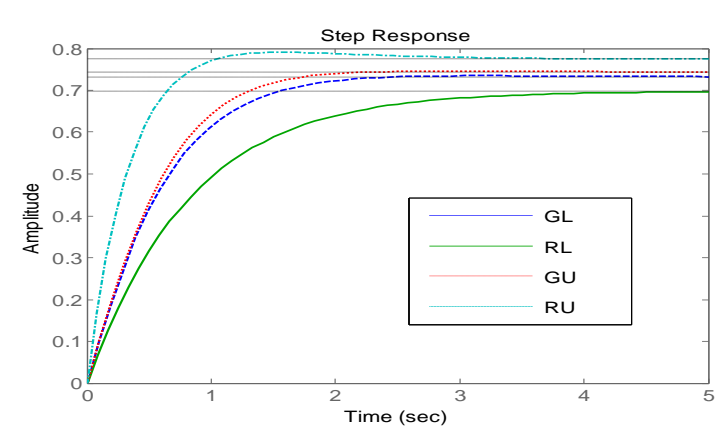

Figure 3: Step Response of Original Model and ROM by Differentiation and Cauer Second Form Method

Method 4: Reduction by differentiation and moment matching method

$$
\begin{aligned}
G_{3}(s) & =\frac{[2,3] s^{2}+[17.5,18.5] s+[15,16]}{[2,3] s^{3}+[17,18] s^{2}+[35,36] s+[20.5,21.5]} \\
G_{3}(s) & =\frac{[0.6977,0.7805]+[0.8139,0.9024] s+[0.093,0.1463] s^{2}}{[1,1]+[1.6279,1.7561] s+[0.7907,0.808] s^{2}+[0.093,0.1463] s^{3}}
\end{aligned}
$$

For getting the second order model, denominator by using differentiation method

Number of times to be differentiated is (n-k) times

$\mathrm{n}=$ order of the higher order system $=3$

$\mathrm{k}=$ order of lower order system $=2$

$D_{2}(s)=[1,1]+[1.0543,1.1707] s+[0.2636,0.2927] s^{2}$

Numerator is reduced by moment matching method

$\left[c_{0}^{-}, c_{0}^{+}\right]=[0.6977,0.7805]$

$\left[c_{1}^{-}, c_{1}^{+}\right]=[-0.5567,-0.2334]$

$\left[c_{2}^{-}, c_{2}^{+}\right]=[-0.2124,0.5724]$

$\left[c_{3}^{-}, c_{3}^{+}\right]=[-0.9349,0.7969]$

$\left[f_{1}^{-}, f_{1}^{+}\right]=\left[\begin{array}{c}{\left[B_{12}^{-}, B_{12}^{+}\right]} \\ {\left[B_{13}^{-}, B_{13}^{+}\right]}\end{array}\right]=\left[\begin{array}{c}{[1.0543,1.1707]} \\ {[0.2636,0.2927]}\end{array}\right]$

$\left[f_{2}^{-}, f_{2}^{+}\right]=\left[\begin{array}{c}{\left[B_{21}^{-}, B_{21}^{+}\right]} \\ {\left[B_{22}^{-}, B_{22}^{+}\right]}\end{array}\right]=\left[\begin{array}{l}{[0.6977,0.7805]} \\ {[0.1789,0.6903]}\end{array}\right]$

$R_{2}(s)=\frac{[0.0111,0.0449] s+[0.0433,0.0508]}{[0.2636,0.2927] s^{2}+[1.0543,1.1707] s+[1,1]}$

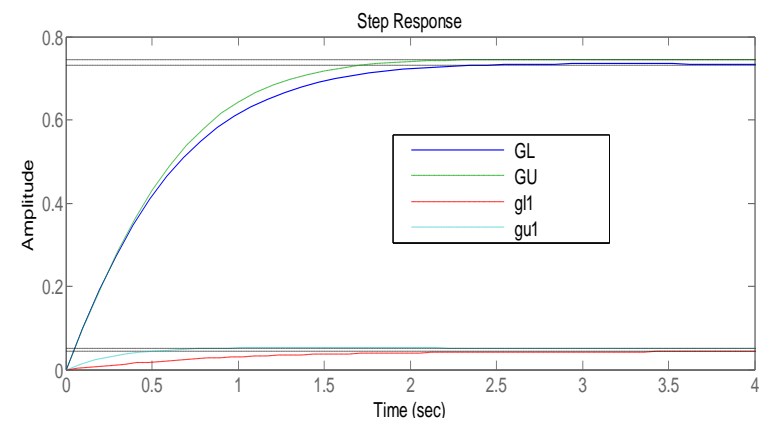

Figure 4: Step Response of Original Model and ROM by

Differentiation and Moment Matching Method

Method 5: Reduction by differentiation and Pade approximation method

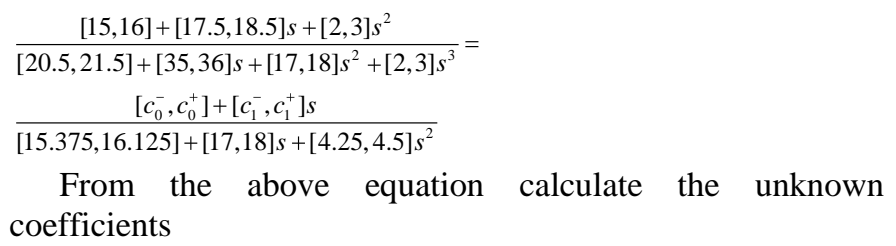

$\left[c_{0}^{-}, c_{0}^{+}\right]=[10.7267,12.5854]$

$\left[c_{1}^{-}, c_{1}^{+}\right]=[3.3018,10.2867]$

The reduced transfer function is

$R_{2}(s)=\frac{[3.30187,10.2867] s+[10.7267,12.5854]}{[4.25,4.5] s^{2}+[17,18] s+[15.375,16.125]}$

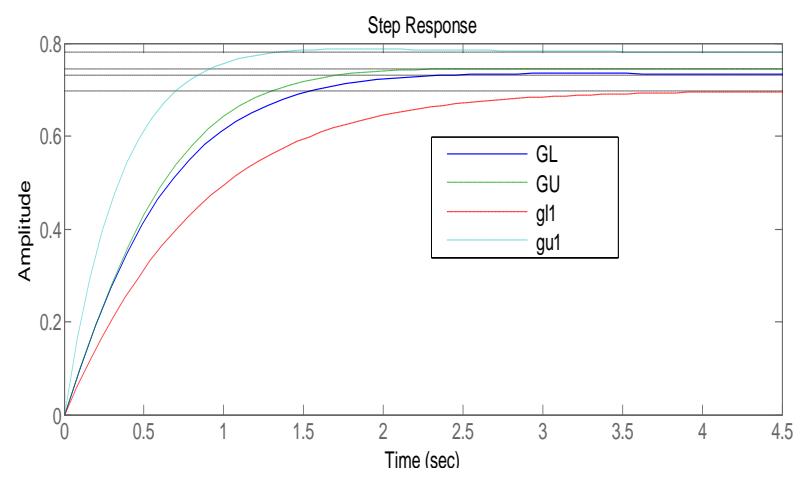

Figure 5: Step Response of Original Model and ROM by Differentiation and Pade Approximation Method

Table 1: Comparison of Reduced Order Models

\begin{tabular}{|l|l|l|}
\hline Method of Order reduction & $\begin{array}{l}\text { ISE for lower } \\
\text { limit }\end{array}$ & $\begin{array}{l}\text { ISE for } \\
\text { upper limit }\end{array}$ \\
\hline Differentiation & 0.0531 & 0.0617 \\
\hline Differentiation and factor division & 0.0094 & 0.0074 \\
\hline $\begin{array}{l}\text { Differentiation and Cauer second } \\
\text { form }\end{array}$ & 0.0094 & 0.0073 \\
\hline $\begin{array}{l}\text { Differentiation and moment } \\
\text { matching }\end{array}$ & 4.1118 & 4.219 \\
\hline $\begin{array}{l}\text { Differentiation and Pade } \\
\text { approximation }\end{array}$ & 0.0094 & 0.0105 \\
\hline G.V.K. Sastry [14] & 0.2256 & 0.0095 \\
\hline
\end{tabular}

From the above figures GL, GU is the higher order lower limit and upper limits transfer function plots. RL and RU for method1, method 2 and method 3 are the reduced order lower limit and upper limit transfer function plots. gl1, gu1 are the reduced order lower limit and upper limit transfer function plots for method4 and method 5 .

From the above mixed methods, differentiation method and cauer second form as resulted in better approximation when compared with other methods.

\section{CONCLUSION}

In this paper new mixed methods are employed for order reduction. The denominator polynomial of reduced model is obtained by using differentiation method. The numerator is obtained by different mixed methods, such as direct truncation 
of differentiation, factor division, Cauer second form, Moment matching method and Pade approximation method. The Moment matching method is not guaranteed to be good method for reduction but is worth a new methodology to obtain a reduced order system. Being not useful here is not enough for its suitability, as when used along with some other methods generates useful and brilliant results. The proposed method guarantees the stability of reduced model if the original system is stable and conceptually simple with better results as compared to existing methods.

\section{ACKNOWLEDGMENT}

Mr.D. Kranthi Kumar gratefully acknowledged to Rajiv Gandhi National Fellowship (UGC), India providing financial assistance in the form of Senior Research Fellowship.

\section{REFERENCES}

[1] M. Aoki, 'Control of Large-Scale Dynamic Systems by Aggregation', IEEE Trans. Autom. Control, 13, 246-253, 1986.

[2] Y. Shamash, 'Stable Reduced Order Models Using Pade Type Approximation', IEEE Trans. Autom. Control, 19, 615-616, 1974.

[3] M.F. Hutton and B. Friedland, 'Routh Approximation for Reducing Order of Linear Time Invariant System', IEEE Trans. Autom. Control, 20, 329-337, 1975.

[4] Y. Shamash, 'Model Reduction Using Routh Stability Criterion and The Pade Approximation', International Journal of Control, 21, 475-484, 1975.

[5] V. Krishnamurthy and V. Seshadri, 'Model Reduction Using Routh Stability Criterion', IEEE Trans. Autom. Control, 23, 729-730, 1978.

[6] K. Glover, 'All Optimal Hankel-norm Approximations of Linear Multivariable Systems and their $L_{\infty}$ Error Bounds', International Journal of Control, 39 (6), 1115-1193, 1984.

[7] N.K. Sinha, and B. Kuszta, 'Modelling and Identification of Dynamic Systems', New York Van Nostrand Reinhold, Ch.8, 133- 163, 1983.

[8] Wan Bai-Wu., 'Linear Model reduction is Using Mihailov Criterion and Pade Approximation Technique', International Journal of Control, 33(6), 1073, 1981.

[9] G. Parmar, 'A Mixed Method for Large-Scale Systems Modelling Using Eigen Spectrum Analysis and Cauer Second Form', IETE Journal of Research, 53, 2, 89-93, 2007.

[10] V. L. Kharitonov, 'Asymptotic Stability of an Equilibrium Position of a Family of Systems of Linear Differential Equations', Differentsial'nye Uravneniya, 14, 2086-2088, 1978.

[11] S.P. Bhattacharyya, 'Robust Stabilization Against Structured Perturbations',(Lecture Notes In Control and Information Sciences). New York: Springer-Verlag, 1987.

[12] B. Bandyopadhyay, O. Ismail, and R. Gorez, 'Routh Pade Approximation for Interval Systems', IEEE Trans. Autom. Control, 39, 2454-2456, 1994.

[13] B. Bandyopadhyay, ' $\gamma-\delta$ Routh Approximations for Interval Systems', IEEE Trans. Autom. Control, 42, 1127-1130, 1997.

[14] G.V.K. Sastry, G.R. Raja Rao and P.M. Rao, 'Large Scale Interval System Modelling Using Routh Approximants', Electronics Letters, 36 (8), 768, 2000.

[15] Y. Dolgin, and E. Zeheb, 'On Routh Pade Model Reduction of Interval Systems', IEEE Trans. Autom. Control, 48 (9), 1610-1612, 2003.

[16] C. Hwang, and S.F. Yang, 'Comments on the Computation of Interval Routh Approximants', IEEE Trans. Autom. Control, 44 (9), 1782-1787, 1999.

[17] Y. Dolgin, 'Author's Reply', IEEE Trans. Autom. Control, 50 (2), 274$275,2007$.

[18] O. Ismail, and B. Bandyopadhyay, 'Model Order Reduction of Linear Interval Systems Using Pade Approximation', IEEE International symposium on circuit and systems, 1995.

[19] G. Saraswathi, 'A Mixed Method for Order Reduction of Interval Systems', International Conference on Intelligent and Advanced Systems, 1042-1046, 2007.

[20] V.P. Singh, and D. Chandra, 'Routh Approximation Based Model Reduction Using Series Expansion of Interval Systems', IEEE
International conference on power, control \& embedded systems (ICPCES), 1, 1-4, 2010

[21] V.P. Singh, and D. Chandra, 'Model Reduction of Discrete Interval System Using Dominant Poles Retention and Direct Series Expansion Method', IEEE $5^{\text {th }}$ International power engineering and optimization conference (PEOCO), 1, 27-30, 2011.

[22] Younseok Choo., 'A Note on Discrete Interval System Reduction via Retention of Dominant Poles', International Journal of Control, Automation, and System, 5 (2), 208-211, 2007.

[23] D. Kranthi Kumar, S.K. Nagar and J.P. Tiwari, "Model order reduction of interval systems using modified Routh approximation and factor division method," $35^{\text {th }}$ National System Conference (NSC), IIT Bhubaneswar, India, 2011.

[24] D. Kranthi kumar, S.K. Nagar and J.P. Tiwari, "Model Order Reduction of Interval Systems Using Mihailov Criterion and Routh Approximations", International Journal of Engineering Science and Technology (IJEST), Vol. 3 No. 7, Pp. 5593-5598, July 2011.

[25] D. Kranthi kumar, S.K. Nagar and J.P. Tiwari, "Model Order Reduction of Interval Systems Using Mihailov Criterion and Factor Division Method", International Journal of Computer Applications (IJCA), Vol. 28- No.11, Pp. 4-8, August 2011.

[26] D. Kranthi kumar, S.K. Nagar and J.P. Tiwari, "Model Order Reduction of Interval Systems Using Routh Approximations and Cauer Second Form", International Journal of Advance Science and Technology (IJAST), August 2011.

[27] D. Kranthi kumar, S.K. Nagar and J.P. Tiwari, "Model Order Reduction of Interval Systems Using Mihailov Criterion and Cauer Second Form", International Journal of Computer Applications, 32(6):17-21, October 2011 .

[28] E. Hansen, 'Interval Arithmetic in Matrix Computations', Part I, SIAM J. Numerical Anal., 308-320, 1965.

[29] P.O. Gutman, C.F. Mannerfelt, and P. Molander, 'Contributions to the Model reduction Problem', IEEE Trans. Autom. Control, 27, 2, 454-455, 1982.

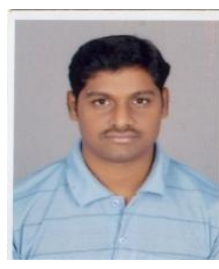

D. Kranthi Kumar was born in Guntur, A.P, India in 1986. He received the degree of B.E from SIR. C. R. Reddy College of Engineering in 2008 and received the degree of M.Tech in Electrical Engineering from IITBHU, Varanasi, in 2010 and presently pursuing Ph.D in Electrical Engineering at IIT-BHU, Varanasi, India. He is life member in System Society of India (SSI), student member in Institute of Engineers (Ireland) and Graduate student member in IEEE. Email:kranthi.kumar.eee08@itbhu.ac.in

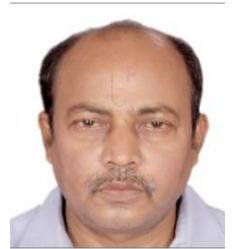

S. K. Nagar was born in Varanasi, India in 1955. He received the degrees of B.Tech and M.Tech from the Institute of Technology, Banaras Hindu University (ITBHU), Varanasi, India In 1976 and 1978; and PhD in Electrical Engineering from University of Roorkee (IIT Roorkee), India in 1991. He is currently Professor in Electrical Engineering at IIT-BHU, Varanasi, India. His main research includes digital control, model order reduction and discrete event systems. He has published many papers in national \& International conferences \& Journals. He is a life member in System Society of India (SSI) and Institute of Engineers (India). E-mail: sknagar.eee@itbhu.ac.in

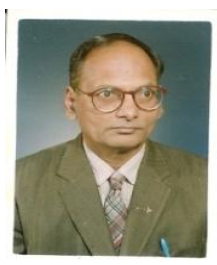

J. P. Tiwari was born in Deoria, U.P, India in 1947. He received the degrees of B.E. and M.E. from University of Roorkee, India in 1968 and 1971. Ph.D in Electrical Engineering from Banaras Hindu University, India in 1991. He is Ex-Professor, at IIT-BHU, Varanasi, India. His main research includes Control systems, Robotics, Instrumentation, Control Systems, Adaptive control. He has published many papers in National \& International conferences and journals. He is a life member in System Society of India (SSI) and Institute of Engineers (India). E-mail: jptewari@bhu.ac.in 